\title{
EXTERNALIDADES SOCIOAMBIENTALES DE LA PRODUCCIÓN DE AGROCOMBUSTIBLES: Nuevos Problemas
}

\author{
Eliana María Noya Díaz \\ Mario de Jesús Zambrano Miranda ${ }^{* *}$
}

La problemática de los agrocombustibles radica en la apropiación del uso de la tierra cuyos impactos ambientales, económicos y en las condiciones de vida de las poblaciones rurales Serán mucho más graves que ahora. (Álvarez, 2008)

\footnotetext{
* Estudiante VI Semestre de Economía.

** Profesor tiempo completo ocasional Programa de Economía de la Universidad de Pamplona, Colombia. Economista, Licenciado en Ciencias Sociales. Especialista en Gestión Pública. E-mail: mariozambrano1883@hotmail.com.
} 


\section{EXTERNALIDADES SOCIOAMBIENTALES DE LA PRODUCCIÓN DE AGROCOMBUSTIBLES: Nuevos Problemas}

\section{RESUMEN}

La aproximación que se hará a continuación sobre la problemática medioambiental y social de los agrocombustibles en Colombia está basado en la reflexión que hizo Margarita Flórez (Flórez, 2008). Según esta autora actualmente en Colombia se está evidenciando una transformación del sector rural enfocada a fortalecer su planificación, industrialización y modernización rediseñada para que funcione con la lógica del modelo de desarrollo económico neoliberal, produciendo iniciativas que solo atienden a determinados intereses agroexportadores y comerciales ,y peor aun teniendo repercusiones en materia ambiental y por supuesto afectando a los agentes vitales en estos procesos: Los pequeños campesinos.

Palabras Claves: Agrocombustibles, modelo de desarrollo, enfoque ambiental.

\begin{abstract}
The approach will be made below on the environmental and social problems of biofuels in Colombia are based on reflection that made Margarita Flórez (Flórez, 2008). According to her in Colombia today is witnessing a transformation of the rural sector focused on strengthening planning, industrialization and modernization redesigned to run on the logic of the neoliberal model of economic development, producing initiatives that serve only special interests and commercial agricultural exporters and worse still having an impact on the environment and of course affecting the vital agents in these processes: small farmers.
\end{abstract}

Key Words: Agro fuels, model development, environmental focus.

\section{Face IssN 1794-9920}

Recepción: Julio de 2010 Revisión: Diciembre de 2010

Aceptación: 2011 
Una de esas iniciativas es la modalidad de utilización de material biológico, especialmente agrícola, como fuente de energía destinado para producir combustibles, comúnmente llamados agrocombustibles o biocombustibles líquidos. En Colombia los principales son el etanol que surge a partir de caña de azúcar y maíz y el agrodiésel basado en palma d aceite y soya entre otros. Estos son impulsados como supuestas soluciones al actual panorama ambiental, energético y agrícola por parte de gobiernos, instituciones y gremios iniciados a nivel mundial y luego a nivel local. Esta nueva alternativa es de especial atención ya que corresponde a un proceso global en crecimiento que relaciona diversas problemáticas por sus repercusiones de carácter ecológico, social, alimentario, económico, energético que no están siendo consideradas para su generación. A demás en el país constituye una preocupación más que afecta el agro colombiano.

Por tal motivo siguiendo la reflexión de Flórez el presente escrito plantea las principales externalidades negativas desde el enfoque ambiental y social que se omiten en el actual crecimiento de la producción de los agrocombustibles que pueden profundizar aún más las causas consideradas para su impulso. En primer lugar se describen los sucesos a nivel internacional y nacional que motivaron a la generación de los agrocombustibles. En segundo lugar se mencionan sus efectos medioambientales bajo el enfoque de ciclo de vida en el uso de la tierra, biodiversidad, el aire y el suelo. Seguido de sus implicaciones sociales como la seguridad alimentaria y campesinado.

\section{CAUSAS DE SU IMPULSO}

A nivel mundial los motivos que tuvieron los gobiernos, entidades y sectores en cuenta para el desarrollo de los agrocombustibles estaban en función de los asuntos energéticos, ambientales y económicos, presentando las ventajas y beneficios pero sin tomar en cuenta las consecuencias. He aquí la importancia de este sector agroindustrial que se caracteriza por la existencia de un gran número de partes involucradas con diversos intereses.

Empezando en el orden energético sus argumentos giran en torno a la preocupación por la seguridad energética mundial que depende de combustibles fósiles como el petróleo. La creciente demanda de consumo está agotando las reservas de este recurso no renovable. Por tanto con la iniciativa de buscar nuevas fuente de energía limpia se encuentra la alternativa de generarla por medio de las materias primas provenientes del campo como maíz, soya, palma aceitera, caña de azúcar, yuca etc. para producir etanol y agrodiésel cuya ventaja en comparación con los combustibles convencionales es que son renovables e inagotables. Además puede disminuir la dependencia del petróleo y los países podrán ser más autosuficientes en sus necesidades energéticas ya que podrán cultivar ellos mismos sus combustibles. 
EXTERNALIDADES SOCIOAMBIENTALES DE

LA PRODUCCIÓN DE AGROCOMBUSTIBLES:

Nuevos Problemas

Pero estas ventajas no son sustentables ya que diversos estudios como los de David Pimentel de Cornell University y Tad Patzek de la Universidad de Berkeley llegan a la conclusión de que para producirse agrocombustibles se necesita más energía de la que se produce, debido al uso intensivo de petróleo para insumos de producción agrícola industrial para plantas de etanol. Las estimaciones reflejan que según el cultivo que se trate se requiere hasta un 45\% más energía fósil que el combustible producido. (Ribeiro, 2007).

Además si el contexto está enfocado especialmente hacia la demanda total de energía, para el año 2030, toda la energía renovable incluidos los agrocombustibles solo constituirán el $9 \%$ del consumo energético mundial, así que el porcentaje restante provendrá de combustibles como el petróleo, carbón y gas natural como indica la grafica $1 .{ }^{1}$

Gráfica 1. Uso de energía global comerciable según tipo de combustible

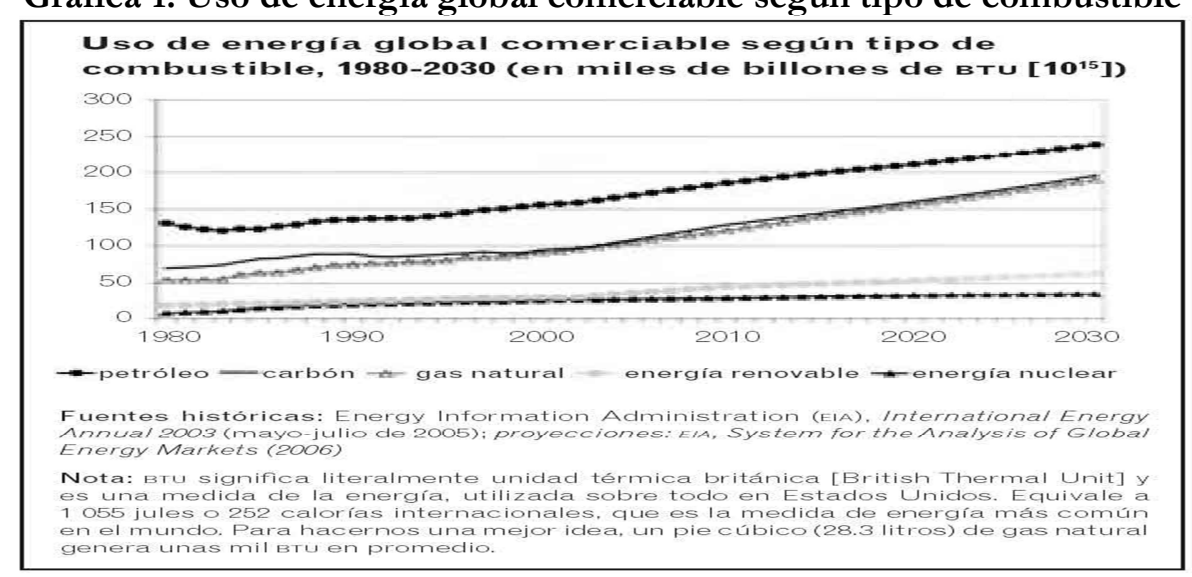

En el contexto ambiental las principales consideraciones se basan en las alternativas a la mitigación al cambio climático, especialmente el calentamiento global causado por la emisión de Gases de efecto invernadero como el dióxido de carbono (Co2), óxido nitroso (NOx), metano (CH4) entre otros. Los principales beneficios del de los agrocombustibles son la reducción de estas emisiones y reducción de contaminantes locales (ciudades). La mayoría de estudios entre esos lo de la FAO mencionan que éstas reducciones en los gases de efecto invernadero que podrían ser del 20 al 60 por ciento en comparación con los combustibles fósiles pero con la gran limitación de que se excluye el carbono en el cambio del uso de la tierra, pero éste factor lamentablemente si influye de manera importante en la producción de los biocombustibles, que al incluirlo

${ }^{1}$ Consultado en http://www.grain.org/article/entries/1150-paremos-la-fiebre-de-los-agrocombustibles 
genera las mayores externalidades negativas tanto ambientales como sociales. Sobre este tema se hablara más adelante.

Por último la motivación relacionada en materia económica se origina en los beneficios de las industrias del sector petrolero, automovilístico, biotecnológico, intermediarias de alimentos y firmas mundiales de inversión que forman parte de una cadena para producir los agrocombustibles. Estos beneficios se acrecientan con las inversiones otorgados por entidades como el Banco Interamericano de Desarrollo, subsidios asignados por los gobiernos para la producción de agrodiésel y etanol y cambios de regulaciones para asegurar mezclas combustibles convencionales en los estos agrocombustibles. Una supuesta ventaja también económica que justifica sus políticas es el acceso que tendrán países pobres a nuevos y exuberantes mercados de exportación. (Grain, 2007).

Regido por estas condiciones el gobierno Colombiano también se ha enfocado en la producción de los agrocombustibles, propiciando su desarrollo y consumo por medio de políticas de subsidio a gremios como el azucarero y de la palma de aceite, mediante expedición de leyes como la 693 de 2001 que estableció que a partir del año 2005 la gasolina colombiana debía tener elementos oxigenado que disminuyeran las emisiones nocivas del medio ambiente, dónde se propone el uso que mezclan $90 \%$ gasolina y $10 \%$ de etanol para cumplir con la norma, entre otros incentivos que han propiciado la acelerada transformación de la agricultura tradicional basada en abastecimiento de alimentos en una agricultura industrial que se trate de adaptar al actual modelo de desarrollo sin considerar las consecuencias ambientales y sociales.

Es más el mismo Gobierno Nacional sostiene que el país tiene ciertas ventajas competitivas en comparación de otros países por su ubicación geográfica que lo hace merecedor de un gran número de zonas tropicales, condiciones climáticas y suelos propicios para la expansión de cultivos como la palma aceitera y la caña de azúcar. En cifras la caña de azúcar ocupaba cerca de 193.423 hectáreas en 2009 y el 95,4\% se produjo en los ingenios y plantas de producción del Valle. Por su parte, en palma de aceite Colombia contaba en 2009 con más de 360.537 hectáreas sembradas distribuidas por zonas así: $37,6 \%$ en la oriental, $30,5 \%$ en la norte, $27,7 \%$ en la central y $4 \%$ en la occidental. (PNUD, 2011).

Entre los posibles aspectos positivos a nivel social y económico se nombra que la implementación de programas de producción de agrocombustibles en el país "puede representar una tabla de salvamento para muchas comunidades rurales amenazadas por los sectores armados y deprimidas por los bajos precios de productos básico a consecuencia de los tratados de libre comercio." (Cardona, 2009). Además crearía nuevos empleos, reduciría la pobreza, impulsaría el crecimiento económico y sustituiría gran parte de las actividades ilícitas realizadas en el campo, lo cual supuestamente 
EXTERNALIDADES SOCIOAMBIENTALES DE

LA PRODUCCIÓN DE AGROCOMBUSTIBLES:

Nuevos Problemas

contribuiría a un mejoramiento en el desarrollo rural y la calidad de vida de los pequeños y medianos productores.

\section{EXTERNALIDADES MADIOAMBIENTALES}

Los impactos negativos en la biodiversidad, suelo, aire y agua de la implementación del sector de agrocombustibles que se dejan a un lado son ocasionados en todas las etapas de producción, pero tiende a dominar los procesos relacionados con el cambio del uso de la tierra y la intensificación dados en la etapa de producción. Para entender más a fondo estas repercusiones en el ambiente diversos estudios se basan en el enfoque del ciclo vital que se trata de un análisis desde la producción agrícola hasta el consumo en los vehículos de transporte. Por la completitud de este método la presentación de las principales externalidades negativas en el medioambiente tomará como referencia un estudio realizado por José Gómez, José Luis Amaniego y Mariana Antoniessen que está basado en este esquema, dónde los impactos ambientales se pueden asociar a las fases de producción agrícola, transformación industrial y consumo. En la gráfica 2 se presentan el esquema general.

Grafica 2: Impactos Ambientales Asociados A Los Biocombustibles.

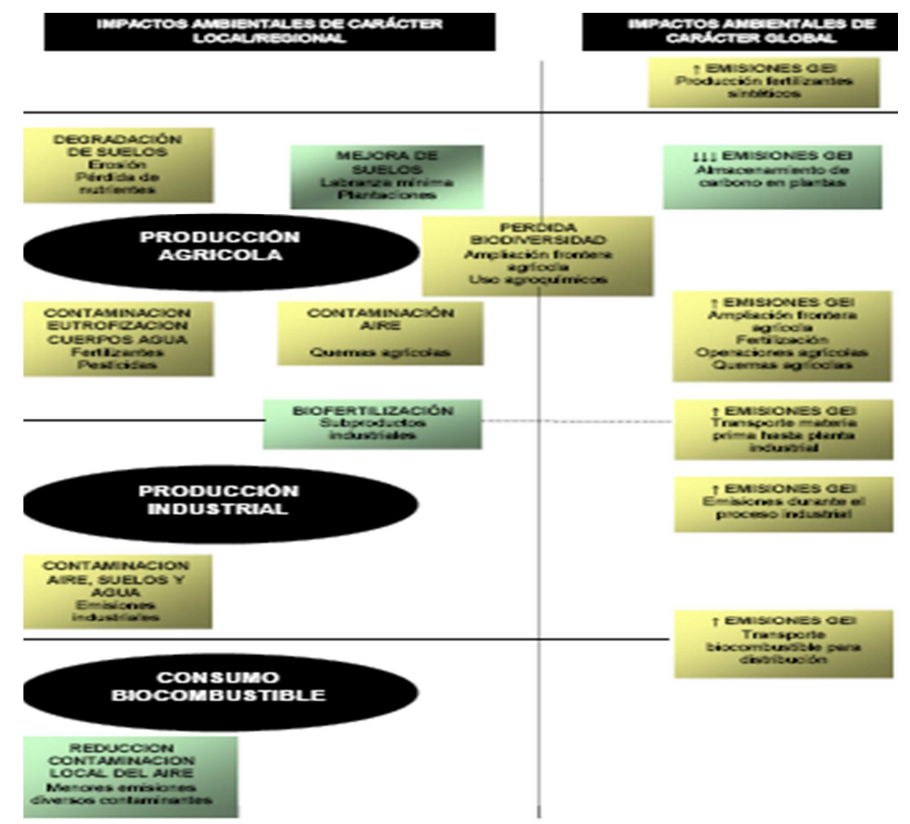

Fuente: Gómez, Samaniego y Antoniessen 2008 


\section{Etapa de producción}

La etapa de producción agrícola abarca diversas situaciones en función del el uso actual de la tierra, del tipo de cultivo escogido como materia prima y de la forma de producción es decir usos de fertilizantes, pesticidas, maquinaria y agua.

Con relación al uso de la tierra, para producir agrocombustibles se parte de la deforestación ya sea por ocupación, tala o quema de bosques. Esto tiene impactos negativos como la pérdida de biodiversidad, erosión y pérdida de materia orgánica, afectación de las fuentes hídricas y especialmente con la quema más liberación de gases de efecto invernadero. La gravedad de estas repercusiones depende de la magnitud de la expansión de los cultivos y de los servicios ambientales que generen los ecosistemas afectados; son tanto locales como globales. A parte de estos daños ambientales, la conversión de áreas naturales tiene implicaciones de tipo alimentario ya que se genera una expansión de la frontera agrícola para cultivar las materias primas reduciendo las tierras destinadas a alimentos básicos. En Colombia actualmente se están convirtiendo bosques tropicales por cultivos de palma aceitera lo cual se ve reflejado en el aumento de los precios de los productos de la canasta familiar.

La fase de producción también genera impactos en el suelo, agua, aire y biodiversidad. En primer lugar los efectos con respecto al suelo son debido a los agroquímicos y la absorción de macro nutrientes. Por ejemplo los fertilizantes producen disminución del oxigeno por el nitrógeno, fósforo y elevación de gases de efecto invernadero. Por el lado de los recursos hídricos en la actualidad la producción de caña de azúcar, aceite de palma y maíz requieren cantidades relativamente elevadas de agua. El aire es afectado por la quema de plantaciones para eliminar residuos vegetales que repercuten en los ecosistemas cercanos. Por último la producción acaba con la biodiversidad silvestre cercana ya que se ve amenazada por la pérdida de hábitat cuando se expande la frontera agrícola.

\section{Etapa de Transformación industrial}

Los principales problemas ambientales entorno a esta etapa hacen referencia a la contaminación del aire por medio de las emisión de óxido nitroso(NOx), dióxido de carbono(Co2), compuestos orgánicos volátiles y al consumo de agua en el proceso industrial en el caso del etanol. Anteriormente el residuo de este de la fase de destilación y deshidratación llamado vinaza generaba graves problemas ambientales, pero actualmente ya es reciclada para beneficio del mismo proceso. Con referencia al biodiesel los problemas ambientales son la generación de residuos líquidos y sólidos con una alta demanda bioquímica de oxígeno (DBO) y la presencia de malos olores.

\section{Etapa del Consumo}

En la etapa del consumo se puede evidenciar una de las razones por las cuales se impulsó el uso de agrocombustibles: La contribución en la mejora calidad del aire en las 
EXTERNALIDADES SOCIOAMBIENTALES DE

LA PRODUCCIÓN DE AGROCOMBUSTIBLES:

Nuevos Problemas

ciudades. Pero su uso es poco significativo si la calidad del combustible convencional es alta y menor es la edad del motor.

Hay que tener en cuenta algo de gran importancia: Las tasas de crecimiento vehicular contrarrestan el efecto de las medidas de mezcla de combustibles. Siguiendo esta afirmación, el país seguirá con los mismos problemas ya que actualmente se está evidenciando un crecimiento en las ventas en el sector automotor, Según el diario El espectador se estima que en el 2012 crezca la venta de vehículos un 10\% con relación al 2010, exactamente 360.000 unidades, lo que indica la poca efectividad que puede llagar a causar la sustitución de gasolina por etanol si sigue creciendo la compra de automóviles.

Visto ya las implicaciones ambientales en cada proceso se llega a la primera conclusión en el ámbito ambiental según Gómez et al. "El principal riesgo ambiental es la ocupación de áreas naturales para el cultivo de materias primas de los agrocombustibles, ya sea de manera directa o bien como efecto de la sustitución del desplazamiento de otros cultivos." Esto se encuentra en la fase de producción. Si bien esto implica perdidas en áreas naturales, biodiversidad, aumento de las emisiones de gases de efecto invernadero por vía cambio uso del suelo y expansión de la frontera agrícola. La magnitud de estos efectos depende de la manera en que se producen y procesan las materias primas, de la escala de producción y especialmente del modo en que influyen en el cambio del uso de la tierra.

Otra conclusión importante a la que llegaron los autores es que si se incorporan los cambios en el eso de la tierra principalmente la deforestación en el análisis del ciclo de vida, las emisiones de gases de invernadero para algunas materias primas y sistemas de producción de agrocombustibles podrían ser mayores incluso que las de los combustibles fósiles, el resultado más sorprendente es el de aproximadamente un 95 por ciento utilizando etanol de maíz, estudio realizado por Searchigeret en el 2008.

Además los agroquímicos empleados en especial los fertilizantes son muy exigentes en energía para su producción y se le suma sus s grandes emisiones de óxido nitroso, un gas de efecto invernaderos más impactante que el CO2. También hay que tener en cuenta que en el transporte de la biomasa provocan emisiones de gases de efecto invernadero dependiendo de la distancia recorrida.

\section{EXTERNALIDADES SOCIALES}

Las implicaciones sociales que giran en torno a la problemática de la ocupación y uso de tierras se manifiestan en la expansión de la frontera agrícola para ampliar los cultivos de materias primas para la producción de los agrocombustibles. Por un lado se pone en 
riesgo la seguridad alimentaria del país por el desplazamiento de cultivos y por otro lado profundiza aun más los problemas de los campesinos e indígenas al ser despojados de sus tierras. Estas consecuencias lo que generan es agudizar más el problema agrario del país. Absalón Machado lo define como:

“... es la carencia de una decisión política de reformar la estructura agraria en función de objetivos de desarrollo y equidad de largo plazo. Es la no consideración del sector agropecuario como un sector estratégico para la sociedad, tanto en términos de seguridad alimentaria como de dominio territorial a través de actividades licitas, y también en calidad del sector que contribuye con externalidades significativas en la conservación del medio ambiente y calidad de vida..." (Machado)

Por tanto afirma que el actual modelo de desarrollo que impulsa la agroindustria no tiene en cuenta la importancia de las agriculturas campesinas e ignora la capacidad que tiene a la hora de desarrollar prácticas para la preservación de los recursos naturales, además lo único que hace es generar nuevos problemas a parte de los que ya se viven en el país. Las actividades agrícolas ejercidas por los pequeños y medianos campesinos pueden ser parte de la solución en la mitigación del cambio climático por medio de la conservación, retención y sustitución de carbono y también pueden establecer sistemas agrícolas más ecológicos que soporten los extremos fenómenos naturales.

En la misma posición aparece la FAO mencionado que en esta época caracterizada por flujos mundiales y globalización, desafíos como la pobreza, el hambre, degradación ambiental, y conflictos; la agricultura no puede seguir planificándose y desarrollándose de manera asilada. Le atribuye ventajas en sus actividades como:

- Garante de la seguridad alimentaria.

- Fuente constante de medios de vida para un gran número de personas.

- Proveedor de servicios ecosistémicos al medio ambiente.

- Usuario más eficiente de energía.

La seguridad alimentaria se ve amenazada por la sustitución de cultivos para productos básicos de alimentación por los cultivos de materias primas como la palma aceitera y caña de azúcar, que necesitan enormes extensiones de tierras para su siembra. Es decir considerando la limitada cantidad de tierras disponibles para agricultura que existe en Colombia, esta nueva demanda de tierra para biomasa, especialmente para agrocombustibles implicará incrementar los monocultivos intensivos. Esto conlleva consecuencias negativas tanto para la población rural como la urbana ya que la implementación de estos escasea la producción de alimentos básicos de la canasta familiar colocando en riesgo el abastecimiento de ellos y por tanto generando una 
EXTERNALIDADES SOCIOAMBIENTALES DE

LA PRODUCCIÓN DE AGROCOMBUSTIBLES:

Nuevos Problemas

presión en el alza de sus precios afectando el costo de vida de la mayoría de la población.

Los más preocupante es que según datos de la OCDE, si Inglaterra plantara para etanol toda su superficie agrícola podría llegar a cubrir sólo el 10 por ciento de su demanda de gasolina, y si la Unión Europea plantara para etanol toda su superficie agrícola, llegaría a sustituir sólo un 15 por ciento de del consumo de gasolina. Para estados Unidos ocurre algo similar, dedicando toda su superficie al cultivo de maíz y soya tan solo cubriría el 12 por ciento de la demanda de combustible.

En Colombia la naturaleza de esta alternativa y algunas de sus consecuencias han ampliado la exclusión social, afectando a comunidades campesinas, indígenas y demás...

\section{En suma a nivel social el conflicto por la renta capitalista de la tierra explica por qué los grandes perdedores en todos los tiempos han sido las comunidades que participan en el sector rural, que cuando no fueron expropiadas en nombre de la modernización, les tocó perder en el reparto del excedente capitalista. Pero de una u otra forma siempre han tenido que pagar por el acceso a una tierra que originalmente era suya. (Álvarez, 2008, p170).}

En general, la promoción actual de los agrocombustibles en Colombia está motivada en el ámbito ambiental por la preocupación a la mitigación del cambio climático teniendo objetivos explícitos la disminución de los gases de efecto invernadero y contaminantes en las ciudades. En el ámbito energético su argumentación se basa en la seguridad energética amenazada por el agotamiento de los combustibles fósiles, por tanto se tiene la intención de generar energía renovable, limpia o verde y por último los beneficios económicos que contraen los sectores involucrados en la producción gracias a los diversos subsidios e inversiones impulsados por el gobierno. Todos estos factores responden a la iniciativa global.

Pero hay evidencias de peso que confirman que todos estos argumentos son insostenibles ya que no consideran y dejan a un lado las externalidades ambientales como la degradación del suelo, las emisiones de gases de efecto invernadero, destrucción de la biodiversidad, bosques, cuencas hidrográficas etc. y las externalidades sociales que afectan principalmente a los pequeños campesinos y a la seguridad alimentaria. Es más si lo que se quiere es mitigar el cambio climático la preproducción y producción de los agrocombustibles generan más emisiones y fuera de eso destruye enormes áreas naturales que proporcionas importantes servicios ambientales.

Todos estos impactos son producto del gran problema de la ocupación de tierras naturales y agrícolas en la que se basa este sector agroindustrial promovido por el modelo de desarrollo económico actual que lo único que hace es transformar el sector 
rural pero profundizando aún más la problemática agraria y ecológica, induciendo a nuevos problemas al no evaluar su verdadera magnitud y potencial en al ámbito ambiental y social.

\section{BIBLIOGRAFÍA}

- Álvarez, P. (2008). Una mirada sobre la transformación del campo: El caso de los agrocombustibles en Colombia. En, La cuestión agraria hoy: Colombia tierra sin campesinos. Bogotá.

- Cardona, C. (2009) Perspectivas de la producción de biocombustibles en Colombia: Contexto latinoamericano y mundial. Manizales.

- El sector automotor seguirá de plácemes en 2012. (2012, Enero 5). El Espectador.

- Flórez, P. (2008). Campo y ambiente. En, La cuestión agraria hoy: Colombia tierra sin campesinos. Bogotá.

- Gómez, J., Samaniego, J. \& Antoniessen, M. (2008). Consideraciones ambientales entorno a lo biocombustibles. Revista Cepal. Serie Medio Ambiente. N 137. Chile.

- Grain, (2007) ¡Paremos la fiebre de los agrocombustibles! Revista biodiversidad.

- Higuera, O., Cristancho, J., Flórez, L. (2007). Biocombustibles y su aplicación en Colombia. Pereira: Universidad Tecnológica de Pereira.

- Machado, A. Tenencia de tierras, problema agrario y conflicto. URL http://www.virtual.unal.edu.co/cursos/humanas/2004945/docs_curso/descargas/2da\%20 sesion/Basica/Absalon\%20Machado.pdf.

- Organización de las Naciones Unidas para la Agricultura y el Desarrollo. (2007). El medio ambiente y la agricultura. Tema 6 del programa provisional.

- Organización de las Naciones Unidas para la Agricultura y el Desarrollo (2008). Biocombustibles: perspectivas, riesgos y oportunidades. En, El estado de la agricultura y alimentación.

- Programa de las Naciones Unidas para el Desarrollo (2011).Informe de Desarrollo Humano. Colombia Rural: Razones para la Esperanza. En, Ocupación y uso del territorio. (pp. 85-90). Bogotá.

- $\quad$ Riveiro, S. (2007). Combustibles Ecológicos- Las crisis propician los negocios. 